\title{
Mid-upper arm circumference in detection of weight-for-height Z-score below -3 in children aged 6-59 months
}

\author{
Umesh Kapil ${ }^{1, *}$, RM Pandey ${ }^{2}$, Rahul Bansal ${ }^{3}$, Bhavana Pant ${ }^{3}$, Amit Mohan Varshney ${ }^{3}$, \\ Chander Prakash Yadav ${ }^{2}$, Shikha Sinha ${ }^{4}$, Neha Sareen ${ }^{1}$ and Harshpal Singh Sachdev ${ }^{4}$ \\ 'Department of Gastroenterology and Human Nutrition, All India Institute of Medical Sciences, New Delhi 110608 , \\ India: ${ }^{2}$ Department of Biostatistics, All India Institute of Medical Sciences, New Delhi, India: ${ }^{3}$ Department of \\ Community Medicine, Subharti Medical College, Meerut, Uttar Pradesh, India: ${ }^{4}$ Department of Pediatrics and \\ Clinical Epidemiology, Sitaram Bhartia Institute of Science and Research, New Delhi, India
}

Submitted 27 January 2017: Final revision received 14 0ctober 2017: Accepted 12 December 2017: First published online 5 February 2018

\begin{abstract}
Objective: To evaluate the predictive ability of mid-upper arm circumference (MUAC) for detecting severe wasting (weight-for-height $Z$-score (WHZ) $<-3$ ) among children aged 6-59 months.

Design: Cross-sectional survey.

Setting: Rural Uttar Pradesh, India.

Subjects: Children ( $n$ 18456) for whom both WHZ ( $n$ 18463) and MUAC were available.

Results: The diagnostic test accuracy of MUAC for severe wasting was excellent (area under receiver-operating characteristic curve $=0.933$ ). Across the lower range of MUAC cut-offs (110-120 mm), specificity was excellent (99.1-99.9\%) but sensitivity was poor (13.4-37.2\%); with higher cut-offs (140-150 mm), sensitivity increased substantially (94.9-98.8\%) but at the expense of specificity (37.6-71.9\%). The optimal MUAC cut-off to detect severe wasting was $135 \mathrm{~mm}$. Although the prevalence of severe wasting was constant at $2 \cdot 2 \%$, the burden of severe acute malnutrition, defined as either severe wasting or low MUAC, increased from 2.46 to $17 \cdot 26 \%$ with cut-offs of $<115$ and $<135 \mathrm{~mm}$, respectively. An MUAC cut-off $<115 \mathrm{~mm}$ preferentially selected children aged $\leq 12$ months (OR $=11 \cdot 8 ; 95 \% \mathrm{CI} 8.4$, 16.6) or $\leq 24$ months $(\mathrm{OR}=23 \cdot 4 ; 95 \%$ CI $12 \cdot 7,43 \cdot 4)$ and girls $(\mathrm{OR}=2 \cdot 2 ; 95 \% \mathrm{CI}$ $1 \cdot 6,3 \cdot 2)$.

Conclusions: Based on important considerations for screening and case detection in the community, modification of the current WHO definition of severe acute malnutrition may not be warranted, especially in the Indian context.
\end{abstract}

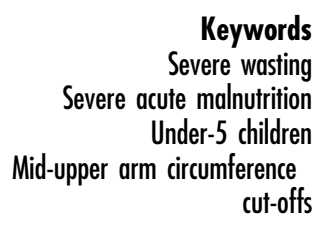

Severe acute malnutrition (SAM) is defined as a low weight-for-height/length (severe wasting; below -3 SD of the median weight-for-height/length of the WHO growth standards), or the presence of visible severe wasting or nutritional oedema, or in children aged 6-59 months, a mid-upper arm circumference (MUAC) of $<115 \mathrm{~mm}^{(1)}$. Management of SAM was estimated to be the most important nutrition intervention to scale up; at $90 \%$ coverage, this mediation could save between 285000 and 482000 lives per year ${ }^{(2)}$. Community-based management is recommended for the overwhelming majority ( $85 \%)$ of children with uncomplicated SAM, including specially formulated diets like ready-to-use therapeutic foods ${ }^{(1,3)}$.

Accurate recording of weight and height/length and their subsequent translation into weight-for-height/length
$Z$-scores can be quite challenging in community settings for health workers. Several large community management of acute malnutrition programmes are therefore increasingly utilizing MUAC to identify SAM. This measurement offers advantages because it requires minimal equipment and is simple, relatively inexpensive and reasonably accurate. However, there can be substantial disagreement between the two anthropometric criteria (weight-for-height/length and MUAC) for identifying children with $\mathrm{SAM}^{(4-9)}$. Thus, remedial suggestions have included an increase in the MUAC cut-off ${ }^{(9)}$ and that the two anthropometric criteria should be used independently to diagnose acute malnutrition $^{(6)}$. Further, the MUAC cut-off of $115 \mathrm{~mm}$ was based predominantly on African data. It is plausible that the association between MUAC and weight-for-height varies in 
South Asian settings; for example, it is now established that for a specific BMI, Asians have a higher percentage of body fat than Europeans ${ }^{(10)}$. The present cross-sectional study was therefore designed to evaluate the predictive ability of various MUAC cut-offs for detecting severe wasting among children aged 6-59 months in a rural area of Meerut District, Uttar Pradesh, India.

\section{Subjects and methods}

The current community-based cross-sectional study was conducted between September 2012 and October 2013 in Meerut District, Uttar Pradesh, India. Uttar Pradesh State had a high prevalence of severe wasting (5.1\%) among children under 5 years old in the National Family Health Survey $3^{(11)}$. Two adjoining rural blocks were identified and their seventy contiguous villages were selected.

The research team members were trained in the methodology of undertaking surveys, recording anthropometry, assessment of age including use of a calendar of local events, and clinical examination for severe visible wasting and bipedal oedema. The training was imparted by two authors (U.K. and R.B.) and experts from the National Institute of Nutrition, Hyderabad, India. Services of village-level health and nutrition functionaries like anganwadi workers, auxiliary nurse midwives and accredited social health activists were utilized for identification of households with children under 5 years of age. However, these functionaries did not participate in any process of data collection including anthropometric measurements.

Children between 6 and 59 months old who were permanent residents of the study area and were not expected to migrate were eligible for inclusion. Children with severe illnesses and physical deformities were excluded. Eligible children likely to be present in each village were estimated from the National Census, 2011. House visits were undertaken to locate them. Subjects who were temporarily unavailable were visited again. 'Mopping-up' of each village was done for 2-3d to ensure inclusion of the maximum number of eligible children. After written informed consent from parents, eligible children were evaluated by the trained research team at local anganwadi centres or health sub-centres or schools. Ethical clearance was obtained from the All India Institute of Medical Sciences.

Sociodemographic profile was recorded on a pre-tested proforma. Exact age was verified through the child's birth certificate, horoscope or hospital record. If such verification was not possible, age was assessed through a local event calendar. Children born on any date of the month were counted as born on the first day of the month. Anthropometric measurements were conducted using standard techniques ${ }^{(12)}$. Length was measured for infants below 24 months of age using a SECA 417 infantometer and height was measured for children 24-59 months old using a SECA 213 digital stadiometer, with a least count of $0 \cdot 1 \mathrm{~cm}$. Weight to the nearest $10 \mathrm{~g}$ was recorded using a SECA 383 digital weighing scale with minimal clothing. MUAC was measured by a SECA 212 fibreglass tape to the nearest $0.1 \mathrm{~cm}$. The weighing scales, infantometer and stadiometer were calibrated on a weekly basis prior to data collection with standard weights (1,2 and $5 \mathrm{~kg}$ ) and a metre rod $(100 \mathrm{~cm})$. Technical errors of measurement for inter-observer and intra-observer variations were below 2\%. Each child was also clinically examined for visible severe wasting and bipedal oedema. WHO reference $Z$-scores were calculated for three anthropometric indices (length/height-for-age, weight-for-age and weight-for-length/height) ${ }^{(13)}$.

Parents or caregivers of SAM children were given appropriate nutritional counselling by the project staff and these subjects were referred to the nearest primary health centre for further management. At the time of conducting the study, there was no dedicated community management of SAM programme in Uttar Pradesh State.

\section{Statistical methods}

Sample size considerations were based on identification of an MUAC cut-off that had a sensitivity of at least $70 \%$, with absolute precision of $5 \%$ and $95 \% \mathrm{CI}$, and a non-response rate of $5 \%$. The Rapid Survey on Children India, conducted in 2013-2014, had documented the prevalence of severe wasting in children under 5 years as $1.5 \%$ (Siddharth Nagar and Sultanpur) and $1.8 \%$ (Sitapur) in neighbouring districts $^{(14)}$. The anticipated prevalence of severe wasting in study area was presumed to be $1 \cdot 8 \%$. We were thus required to approach 19400 subjects for enrolling 336 severely wasted participants; however, we approached 19449 children.

Data analysis was performed using the statistical software packages IBM SPSS Statistics version 20.0 and Stata version 12.0. The receiver-operating characteristic (ROC) curve, sensitivity, specificity, positive and negative predictive values, and positive and negative likelihood ratios were calculated for identification of severe wasting with various MUAC cut-offs. Multiple regression and logistic regression analyses were conducted to evaluate the associations between MUAC and age and sex of the child.

\section{Study oversight}

The study was approved by the Government of India and the Uttar Pradesh State Government. Oversight was provided by the National Research Alliance for SAM established by the Indian Government. An independent institution (Clinical Development Services Agency) periodically audited the study and provided recommendations.

\section{Results}

Among the 19449 children approached through houseto-house survey, 278 were excluded due to deformities 
while 186 were not within the age range of 6-59 months. Of the remaining 18985 children, length/height was available for 18595, weight for 18474 and MUAC for 18499 . We further excluded eleven children from the data set because of unacceptable $Z$-scores ( $n$ 2), visible severe wasting ( $n$ 8) and bipedal oedema ( $n 1)$. The final data set for analysis comprised 18456 children for whom both weight-for-height $Z$-score (WHZ; $n$ 18463) and MUAC were available. There were 9858 (53.4\%) boys. The mean (sD) age was $2.72(1.29)$ years; children aged <12, 12-24 and $>24$ months comprised $11.9,22.3$ and $65.8 \%$ of the sample, respectively.

Severe wasting was observed in $2.2 \%$ of participants (409/18463; $95 \%$ CI 2.02, 2.44\%). The prevalence declined with increasing age $(P=0.001)$, with estimates in age groups of $6-11,12-23,24-35,36-47$ and 48-59 months being $4 \cdot 2,3 \cdot 3,1 \cdot 6,1.2$ and $1.7 \%$, respectively. Severe stunting (height-for-age $Z$-score $<-3$ ) was documented in $16.2 \%$ and moderate stunting (height-forage $Z$-score $\geq-3$ to -2 ) in $29 \cdot 1 \%$ of 17786 children for whom the exact date of birth was available. The prevalence of severe stunting (WHZ $<-3$ ) in age groups 6-11, 12-23, 24-35, 36-47 and 48-59 months was 11.9, 19.3, $20 \cdot 5,16 \cdot 2$ and $11.0 \%$, respectively.

Figure 1 depicts the ROC curve, whereas Table 1 summarizes the diagnostic test accuracies for varying MUAC cut-offs with severe wasting as the 'gold standard'. The area under the ROC curve was 0.933. Across the lower range of MUAC cut-offs (110-120 mm), specificity was excellent (99.1-99.9\%) but sensitivity was poor (13.4-37.2\%); with higher cut-offs (140-150 mm), sensitivity increased substantially (94.9-98.8\%) but at the expense of specificity (37.6-71.9\%). With $<115 \mathrm{~mm}$ as MUAC cut-off, $76.8 \%$ of subjects with severe wasting were missed but only $0.3 \%$ of screened negatives were severely wasted. The optimal MUAC cut-off to detect severe wasting was $135 \mathrm{~mm}$; only $11.7 \%$ of subjects with severe wasting were missed while $15.4 \%$ of screened negatives were severely wasted.

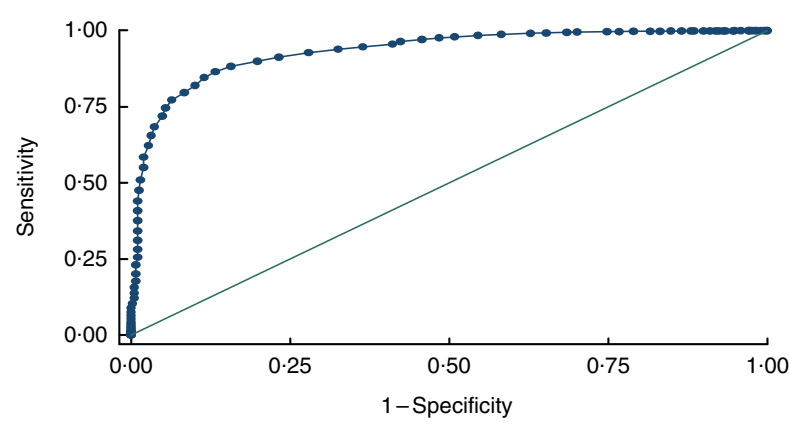

Fig. 1 Receiver-operating characteristic (ROC) curve plotting the sensitivity and specificity of different mid-upper arm circumference cut-offs for detecting severe wasting (weight-for-height $Z$-score $<-3$ as the 'gold standard) among children ( $n$ 18456) aged 6-59 months, rural Uttar Pradesh, India, September 2012October 2013 (area under the ROC curve $=0.9334$ )
Table 2 summarizes the prevalence of SAM using various combinations of MUAC cut-offs and $\mathrm{WHZ}<-3$. The prevalence of severe wasting was constant at $2 \cdot 2 \%$. However, the prevalence of MUAC screened positive increased substantially with higher cut-offs, with values of $0.76 \%$ and $17.00 \%$ for $<115$ and $<135 \mathrm{~mm}$, respectively. The corresponding burden of SAM, defined as either severe wasting or low MUAC, increased from $2 \cdot 46$ to $17 \cdot 26 \%$ with cut-offs of $<115$ and $<135 \mathrm{~mm}$, respectively.

MUAC was higher in older children and boys, even after adjustment for the weight-for-height status $(P<0 \cdot 001)$. An MUAC cut-off of $<115 \mathrm{~mm}$ preferentially selected children aged $\leq 12$ months $(\mathrm{OR}=11.8 ; 95 \%$ CI $8.4,16 \cdot 6)$ or $\leq 24$ months $(\mathrm{OR}=23.4 ; 95 \%$ CI $12.7,43.4)$ and girls $(\mathrm{OR}=2 \cdot 2 ; 95 \% \mathrm{CI} 1 \cdot 6,3 \cdot 2)$. These OR were lower with an MUAC cut-off of $<135 \mathrm{~mm}$ : children aged $\leq 12$ months $(\mathrm{OR}=5 \cdot 0 ; 95 \% \mathrm{CI} 4 \cdot 5,5 \cdot 5)$ or $\leq 24$ months (OR $=6 \cdot 0 ; 95 \%$ CI $5 \cdot 5,6 \cdot 5)$.

\section{Discussion}

The diagnostic test accuracy of MUAC for severe wasting was excellent (area under ROC curve $=0.933$ ). However, at lower MUAC cut-offs (110-120 mm), specificity was excellent but sensitivity was poor; while with higher cut-offs (140-150 mm), sensitivity was excellent but specificity was poor. The optimal MUAC cut-off to detect severe wasting was $135 \mathrm{~mm}$. Although the prevalence of severe wasting was constant at $2 \cdot 2 \%$, the SAM burden, defined as either severe wasting or low MUAC, increased from $2 \cdot 46$ to $17 \cdot 26 \%$ with cut-offs of $<115$ and $<135 \mathrm{~mm}$, respectively.

Our data reaffirm earlier observations ${ }^{(5,6,9,15-17)}$ that MUAC $<115 \mathrm{~mm}$ is not the optimal cut-off to detect severe wasting among children aged between 6 months and 5 years. The optimal cut-off in the current community-based data set is similar to that found in earlier analyses from Cambodia $(133 \mathrm{~mm})^{(9)}$ and ten mostly African countries $(<135 \mathrm{~mm})^{(17)}$. This suggests that there are no noteworthy ethnic or geographical differences in the predictive ability of MUAC cut-offs for severe wasting. Even after accounting for weight-for-height status, an MUAC $<115 \mathrm{~mm}$ preferentially identified children aged $\leq 12$ months and girls. This finding is in consonance with the contention that MUAC $<115 \mathrm{~mm}$ and $\mathrm{WHZ}<-3$ identify a distinctly different set of children with malnutrition, with little overlap between the two indicators ${ }^{(9,18)}$. It is hypothesized that the two indicators measure different aspects of body composition, reflecting perhaps different categories of malnutrition ${ }^{(9)}$. Earlier studies in children showed that weight-for-height cannot discriminate between fat and lean body mass ${ }^{(19)}$ whereas MUAC is strongly related to fat mass but poorly related to fat-free mass or overall weight $^{(20)}$. Unfortunately, our data set precludes an examination of this hypothesis. 
Table 1 Diagnostic test accuracy measures for varying cut-offs of mid-upper arm circumference (MUAC) in predicting severe wasting (weight-for-height $Z$-score $<-3$ as the 'gold standard') among children ( $n$ 18 456) aged 6-59 months, rural Uttar Pradesh, India, September 2012-October 2013

\begin{tabular}{|c|c|c|c|c|c|c|c|c|c|c|c|c|c|}
\hline \multirow{2}{*}{$\begin{array}{l}\text { MUAC cut- } \\
\text { off (mm) }\end{array}$} & \multicolumn{2}{|c|}{ Sensitivity (\%) } & \multicolumn{2}{|c|}{ Specificity (\%) } & \multicolumn{2}{|c|}{$\begin{array}{l}\text { Positive likelihood } \\
\text { ratio }\end{array}$} & \multicolumn{2}{|c|}{$\begin{array}{c}\text { Negative } \\
\text { likelihood ratio }\end{array}$} & \multicolumn{2}{|c|}{$\begin{array}{l}\text { Positive } \\
\text { predictive value } \\
(\%)\end{array}$} & \multicolumn{2}{|c|}{$\begin{array}{c}\text { Negative } \\
\text { predictive value } \\
(\%)\end{array}$} & \multirow{2}{*}{$\begin{array}{l}\text { Post-test } \\
\text { odds }\end{array}$} \\
\hline & Mean & $95 \% \mathrm{Cl}$ & Mean & $95 \% \mathrm{Cl}$ & Mean & $95 \% \mathrm{Cl}$ & Mean & $95 \% \mathrm{Cl}$ & Mean & $95 \% \mathrm{Cl}$ & Mean & $95 \% \mathrm{Cl}$ & \\
\hline$<110$ & $13 \cdot 4$ & $10 \cdot 3,17 \cdot 1$ & 99.9 & $99.9,100$ & $186 \cdot 7$ & $102 \cdot 8,338 \cdot 9$ & 0.87 & $0.83,0.90$ & 80.9 & $70 \cdot 0,88 \cdot 5$ & $98 \cdot 1$ & $98 \cdot 0,98 \cdot 1$ & 4.24 \\
\hline$<115$ & $23 \cdot 2$ & $19 \cdot 2,27 \cdot 6$ & 99.7 & $99 \cdot 7,99 \cdot 8$ & $91 \cdot 1$ & $65 \cdot 0,127 \cdot 8$ & 0.77 & $0.73,0.81$ & $67 \cdot 4$ & $59 \cdot 6,74 \cdot 3$ & $98 \cdot 3$ & $98 \cdot 2,98 \cdot 4$ & $2 \cdot 07$ \\
\hline$<120$ & $37 \cdot 2$ & $32 \cdot 5,42 \cdot 0$ & $99 \cdot 1$ & $98 \cdot 9,99 \cdot 2$ & 41.4 & $33 \cdot 9,50 \cdot 5$ & 0.63 & $0.59,0.68$ & $48 \cdot 4$ & $43 \cdot 5,53 \cdot 4$ & $98 \cdot 6$ & $98 \cdot 5,98 \cdot 7$ & 0.94 \\
\hline$<125$ & $54 \cdot 3$ & $49 \cdot 3,59 \cdot 2$ & $97 \cdot 2$ & $96 \cdot 9,97 \cdot 4$ & $19 \cdot 1$ & $16 \cdot 8,21 \cdot 6$ & 0.47 & $0.42,0.52$ & $30 \cdot 2$ & $27 \cdot 6,32 \cdot 8$ & 98.9 & $98 \cdot 8,99 \cdot 1$ & 0.43 \\
\hline$<130$ & 71.9 & $67 \cdot 3,76 \cdot 2$ & $92 \cdot 8$ & $92 \cdot 4,93 \cdot 2$ & $10 \cdot 0$ & $9 \cdot 2,10 \cdot 8$ & 0.30 & $0.26,0.35$ & $18 \cdot 4$ & $17 \cdot 3,19 \cdot 7$ & $99 \cdot 3$ & $99 \cdot 2,99 \cdot 4$ & 0.23 \\
\hline$<131$ & 76.5 & $72 \cdot 1,80 \cdot 5$ & $91 \cdot 3$ & $90 \cdot 9,91 \cdot 7$ & 8.8 & $8 \cdot 2,9.4$ & 0.26 & $0.22,0.31$ & $16 \cdot 6$ & $15 \cdot 6,17 \cdot 6$ & 99.4 & $99.3,99.5$ & 0.20 \\
\hline$<132$ & 79.9 & $75 \cdot 7,83 \cdot 7$ & 89.9 & $89 \cdot 5,90 \cdot 4$ & 7.9 & $7.4,8.5$ & 0.22 & $0.18,0.27$ & $15 \cdot 2$ & $14 \cdot 4,16 \cdot 1$ & 99.5 & $99 \cdot 4,99 \cdot 6$ & 0.18 \\
\hline$<133$ & 84.1 & $80 \cdot 2,87 \cdot 5$ & $88 \cdot 3$ & $87 \cdot 8,88 \cdot 7$ & $7 \cdot 2$ & $6 \cdot 8,7 \cdot 6$ & 0.18 & $0.14,0.22$ & $14 \cdot 0$ & $13 \cdot 3,14 \cdot 7$ & $99 \cdot 6$ & $99 \cdot 5,99 \cdot 7$ & $0 \cdot 16$ \\
\hline$<134$ & $86 \cdot 5$ & $82 \cdot 9,89 \cdot 7$ & 86.5 & $86 \cdot 0,87 \cdot 0$ & $6 \cdot 4$ & $6 \cdot 1,6 \cdot 8$ & $0 \cdot 16$ & $0.12,0.20$ & $12 \cdot 7$ & $12 \cdot 1,13 \cdot 3$ & $99 \cdot 6$ & $99 \cdot 5,99 \cdot 7$ & 0.15 \\
\hline$<135$ & $88 \cdot 3$ & $84 \cdot 7,91 \cdot 2$ & $84 \cdot 6$ & $84 \cdot 1,85 \cdot 1$ & $5 \cdot 7$ & $5 \cdot 5,6 \cdot 0$ & 0.14 & $0.11,0.18$ & 11.5 & $11 \cdot 0,12 \cdot 0$ & $99 \cdot 7$ & $99 \cdot 6,99 \cdot 8$ & 0.13 \\
\hline$<136$ & 89.7 & $86 \cdot 4,92 \cdot 5$ & $82 \cdot 0$ & $81 \cdot 4,82.5$ & $5 \cdot 0$ & $4 \cdot 8,5 \cdot 2$ & 0.13 & $0.09,0.17$ & $10 \cdot 1$ & $9 \cdot 7,10 \cdot 6$ & $99 \cdot 7$ & $99.6,99.8$ & 0.11 \\
\hline$<137$ & 91.4 & $88 \cdot 3,94.0$ & $79 \cdot 7$ & $79 \cdot 1,80 \cdot 3$ & 4.5 & $4 \cdot 3,4 \cdot 7$ & 0.11 & $0.08,0.15$ & $9 \cdot 3$ & $8.9,9.6$ & $99 \cdot 8$ & $99 \cdot 7,99 \cdot 8$ & $0 \cdot 10$ \\
\hline$<138$ & 93.4 & $90 \cdot 5,95 \cdot 6$ & $77 \cdot 2$ & $76 \cdot 6,77 \cdot 8$ & $4 \cdot 1$ & $3 \cdot 9,4 \cdot 3$ & 0.09 & $0.06,0.12$ & 8.5 & $8 \cdot 2,8 \cdot 8$ & 99.8 & $99 \cdot 7,99 \cdot 8$ & 0.09 \\
\hline$<139$ & 94.4 & $91 \cdot 7,96 \cdot 4$ & 74.7 & $74 \cdot 0,75 \cdot 3$ & 3.7 & $3 \cdot 6,3 \cdot 9$ & 0.08 & $0.05,0.11$ & $7 \cdot 8$ & $7.5,8.0$ & 99.8 & $99 \cdot 7,99 \cdot 9$ & 0.08 \\
\hline$<140$ & 94.9 & $92 \cdot 3,96 \cdot 8$ & 71.9 & $71 \cdot 3,72 \cdot 6$ & 3.4 & $3.3,3.5$ & 0.07 & $0.05,0.11$ & $7 \cdot 1$ & $6 \cdot 9,7 \cdot 3$ & $99 \cdot 8$ & $99 \cdot 8,99 \cdot 9$ & 0.08 \\
\hline$<145$ & 97.8 & $95.9,99.0$ & $55 \cdot 1$ & $54 \cdot 4,55 \cdot 8$ & $2 \cdot 2$ & $2 \cdot 1,2 \cdot 2$ & 0.04 & $0.02,0.08$ & 4.7 & $4 \cdot 6,4 \cdot 8$ & 99.9 & $99.8,99.9$ & 0.05 \\
\hline$<150$ & 98.8 & $97 \cdot 2,99 \cdot 6$ & $37 \cdot 6$ & $36 \cdot 9,38 \cdot 4$ & 1.6 & $1 \cdot 6,1 \cdot 6$ & 0.03 & $0.01,0.08$ & 3.5 & $3.4,3.5$ & 99.9 & $99 \cdot 8,100$ & 0.04 \\
\hline
\end{tabular}

Table 2 Prevalence of severe acute malnutrition using various combinations of mid-upper arm circumference (MUAC) cut-offs and weight-for-height $Z$-score (WHZ) $<-3$ in the surveyed population of children ( $n$ 18456) aged 6-59 months, rural Uttar Pradesh, India, September 2012-October 2013

\begin{tabular}{|c|c|c|c|c|c|c|}
\hline \multirow[b]{2}{*}{ MUAC cut-off (mm) } & \multicolumn{2}{|c|}{ Using only MUAC cut-off } & \multicolumn{2}{|c|}{ Using only $\mathrm{WHZ}<-3$} & \multicolumn{2}{|c|}{ Using MUAC cut-off or $\mathrm{WHZ}<-3$} \\
\hline & $n$ & $\%$ & $n$ & $\%$ & $n$ & $\%$ \\
\hline$<110$ & 68 & 0.37 & 409 & $2 \cdot 22$ & 422 & $2 \cdot 29$ \\
\hline$<115$ & 141 & 0.76 & 409 & $2 \cdot 22$ & 455 & 2.46 \\
\hline$<120$ & 314 & 1.70 & 409 & $2 \cdot 22$ & 571 & 3.09 \\
\hline$<125$ & 736 & 3.99 & 409 & 2.22 & 923 & 5.00 \\
\hline$<130$ & 1595 & 8.64 & 409 & $2 \cdot 22$ & 1710 & $9 \cdot 27$ \\
\hline$<134$ & 2781 & 15.07 & 409 & 2.22 & 2836 & $15 \cdot 37$ \\
\hline$<135$ & 3138 & 17.00 & 409 & $2 \cdot 22$ & 3186 & $17 \cdot 26$ \\
\hline$<140$ & 5453 & 29.55 & 409 & $2 \cdot 22$ & 5474 & 29.66 \\
\hline$<145$ & 8500 & 46.06 & 409 & $2 \cdot 22$ & 8509 & $46 \cdot 11$ \\
\hline$<150$ & 11657 & $63 \cdot 16$ & 409 & $2 \cdot 22$ & 11662 & 63.19 \\
\hline
\end{tabular}

\section{Strengths and limitations}

The present community-based study was conducted on a representative and large sample size with good quality control and monitoring. However, the following limitations merit consideration: (i) age approximation was necessitated in a small proportion as exact date of birth was not verifiable; (ii) data collection had to be discontinued for a short period (January 2013) due to severe winter; and (iii) prospective mortality outcomes were not evaluated for the cohort because of ethical considerations. However, these limitations are unlikely to bias the association between weight-for-height and MUAC.

\section{Implications for policy}

The prime objective of identifying children with SAM is to save their lives by enrolling them in a community management of acute malnutrition programme ${ }^{(1,3)}$. Ideally, analyses to define SAM cut-offs should identify the optimal combination of MUAC and weight-for-height using mortality as outcome; however, ethical considerations preclude this in the current era. Therefore, we have to rely (among other aspects) on earlier evidence using mortality as outcome ${ }^{(21,22)}$, suggested key properties for screening and case detection in the community ${ }^{(23)}$, congruence with recent related international guidelines ${ }^{(24)}$ and logistical considerations. The most consistent evidence across studies is that weight-for-height is a less effective predictor of mortality than MUAC in community settings ${ }^{(21,22)}$. Further, MUAC alone had better predictive ability than either of the two possible combinations of WHZ $<-3$ and/or MUAC $\leq 115 \mathrm{~mm}^{(22)}$. For screening and case detection in the community, simplicity and high specificity are desirable properties ${ }^{(23)}$. A high specificity 
would also be in tune with the recent WHO guidelines ${ }^{(24)}$ which recommend 'not to provide formulated supplementary foods on a routine basis to children who are moderately wasted'. Based on the above considerations, the following possibilities, with their attendant pros and cons, deserve deliberation for reaching a programmatic consensus, especially in the Indian context.

Using the optimal MUAC cut-off to increase the sensitivity for detecting severe wasting, a child with SAM could be identified using MUAC $<135 \mathrm{~mm}$ or WHZ $<-3$. However, this enhances the case load sevenfold (2.46 to $17 \cdot 26 \%$ ), creating immense logistical and resource challenges. Using the simpler measure, MUAC $<135 \mathrm{~mm}$ alone (17\%), also presents similar difficulties. A two-step model has been suggested ${ }^{(9)}$. MUAC $<135 \mathrm{~mm}$ could be used for screening at community level, followed by MUAC and weight-for-height measurements at a primary health care unit, with both indicators used independently to diagnose SAM and treatment being initiated when either MUAC $<115 \mathrm{~mm}$ or $\mathrm{WHZ}<-3$. Apart from substantially increasing the logistical requirements, the high false positives - six children will be turned away for every child treated - are likely to undermine confidence in the programme ${ }^{(9)}$.

If specificity is the overriding consideration, then a modification in the current WHO definition of SAM may not be warranted. However, accurate recording of length or height in community settings in low- and middle-income countries poses considerable challenges and many national programmes prefer to use MUAC alone for identification of SAM. Cut-offs of 115 or $120 \mathrm{~mm}$ offer an excellent combination of specificity and logistical requirements based on SAM burden (0.76-1.7\%). Additional advantages include simplicity and feasibility of measurement, and preferential identification of children below 12 months of age and girls, which probably enhances the risk prediction for mortality.

\section{Conclusion}

In conclusion, the diagnostic test accuracy of MUAC for severe wasting was excellent (area under ROC curve $=0.933$ ). The optimal MUAC cut-off to detect severe wasting was $135 \mathrm{~mm}$. Although the prevalence of severe wasting was constant at $2 \cdot 2 \%$, the SAM burden, defined as either severe wasting or low MUAC, increased from 2.46 to $17.26 \%$ with cut-offs $<115$ and $<135 \mathrm{~mm}$, respectively. Based on important considerations for screening and case detection in the community, modification of the current WHO definition of SAM may not be warranted, especially in the Indian context.

\section{Acknowledgements}

Acknowledgements: The authors are grateful to the Clinical Development Services Agency (CDSA) team at the
Department of Biotechnology, Government of India for providing supportive supervision and monitoring the quality of the survey; and the Steering Committee and Technical Advisory Group of the National SAM Alliance (consisting of the Ministry of Health and Family Welfare, Department of Biotechnology, Indian Council of Medical Research) for their technical support and guidance. Financial support: The authors are extremely grateful to the Indian Council of Medical Research for providing the financial grant for conducting this study (sanctioned vide letter number ICMR 5/97/506/2011-NUT). The funder had no role in the design, analysis or writing of this article. Conflict of interest: The authors declare no conflicts of interest. Authorship: U.K.: concept and design of the study; acquisition, analysis and interpretation of the data; drafted the manuscript and final approval of the manuscript. R.M.P.: design of the work; acquisition and analysis of the data; final approval of the manuscript. R.B.: design of the work; acquisition of the data; final approval of the manuscript. B.P.: design of the work; final approval of the manuscript. A.M.V.: design of the work; final approval of the manuscript. C.P.Y.: data analysis. S.S.: data analysis; interpretation of data. N.S.: interpretation of data; drafted the manuscript. H.S.S.: concept and design of the study; acquisition, analysis and interpretation of the data; drafted the manuscript and final approval of the manuscript. Ethics of human subject participation: Ethical clearance for the study was obtained from the ethics committees of the All India Institute of Medical Sciences, New Delhi, India and Subharti Medical College, Meerut, India. Written informed consent was taken from the parent/guardian of each eligible child.

\section{References}

1. World Health Organization (2013) Guideline: Updates on the Management of Severe Acute Malnutrition in Infants and Children. Geneva: WHO.

2. Bhutta ZA, Das JK, Rizvi A et al.; The Lancet Nutrition Interventions Review Group and The Maternal and Child Nutrition Study Group (2013) Evidence-based interventions for improvement of maternal and child nutrition: what can be done and at what cost? Lancet 382, 452-477.

3. Lenters LM, Wazny K, Webb P et al. (2013) Treatment of severe and moderate acute malnutrition in low- and middle-income settings: a systematic review, meta-analysis and Delphi process. BMC Public Health 13, Suppl. 3, S23.

4. Walters T, Sibson V \& McGrath M (2012) Mid Upper Arm Circumference and Weight-for-Height Z-score as indicators of severe acute malnutrition: a consultation of operational agencies and academic specialists to understand the evidence, identify knowledge gaps and to inform operational guidance. http://www.cmamforum.org/Pool/Resources/ MUAC-WFH-Report-ENN-2013.pdf (accessed July 2016).

5. Roberfroid D, Huybregts L, Lachat C et al. (2015) Inconsistent diagnosis of acute malnutrition by weight-forheight and mid-upper arm circumference: contributors in 16 cross-sectional surveys from South Sudan, the Philippines, Chad, and Bangladesh. Nutr J 14, 86.

6. Grellety E \& Golden MH (2016) Weight-for-height and mid-upper arm circumferences should be used 
independently to diagnose acute malnutrition: policy implications. BMC Nutr 2, 10.

7. Guevarra E, Norris A, Guerrero S et al.2012) Assessment of coverage of community-based management of acute malnutrition. CMAM Forum Technical Brief no. 1. http://s3. ennonline.net/attachments/2276/Coverage-and-CMAM-2012-v2sept2014.pdf (accessed September 2016).

8. Ali E, Zachariah R, Shams Z et al. (2013) Is mid-upper arm circumference alone sufficient for deciding admission to a nutritional programme for childhood severe acute malnutrition in Bangladesh? Trans R Soc Trop Med Hyg 107, 319-323.

9. Laillou A, Prak S, de Groot R et al. (2014) Optimal screening of children with acute malnutrition requires a change in current WHO guidelines as MUAC and WHZ identify different patient groups. PLoS One 9, e101159.

10. WHO Expert Consultation (2004) Appropriate body mass index for Asian populations and its implications for policy and intervention strategies. Lancet 363, 157-163.

11. International Institute for Population Sciences \& Macro International (2007) National Family Health Survey (NFHS-3), 2005-2006; India. Volume I. Mumbai: IIPS.

12. World Health Organization (1995) Physical Status: The Use and Interpretation of Anthropometry. Report of a WHO Expert Committee. WHO Technical Report Series no. 854. Geneva: WHO.

13. World Health Organization (2006) The WHO Child Growth Standards. http://www.who.int/childgrowth/standards/en/ (accessed September 2016).

14. Ministry of Women and Child Development, Government of India (2016) Rapid Survey on Children 2013-2014. India Fact Sheet. http://wcd.nic.in/sites/default/files/RSOC\%20FACT\% 20SHEETS\%20Final.pdf (accessed April 2016).

15. Dasgupta R, Sinha D, Jain SK et al. (2013) Screening of SAM in the community: is MUAC a 'simple tool'? Indian Pediatr 50, 154-155.
16. Kumar R, Aggarwal AK \& Iyenger S (1995) Nutritional status of children: validity of mid-upper arm circumference for screening undernutrition. Indian Pediatr 33, 189-196.

17. Fernandez MA, Delchevalerie P \& Van Herp M (2010) Accuracy of MUAC in the detection of severe wasting with the new WHO growth standards. Pediatrics 126, e195-e201.

18. Berkley J, Mwangi I, Griffiths K et al. (2005) Assessment of severe malnutrition among hospitalized children in rural Kenya: comparison of weight for height and mid upper arm circumference. JAMA 294, 591-597.

19. Wells JC, Fewtrell MS, Williams JE et al. (2006) Body composition in normal weight, overweight and obese children: matched case control analyses of total and regional tissue masses, and body composition trends in relation to relative weight. Int J Obes (Lond) 30, 1506-1513.

20. Chomtho S, Fewtrell MS, Jaffe A et al. (2006) Evaluation of arm anthropometry for assessing pediatric body composition: evidence from healthy and sick children. Pediatr Res 59, 860-865.

21. Pelletier DL (1994) The relationship between child anthropometry and mortality in developing countries: implications for policy, programs and future research. J Nutr 124, 10 Suppl., 2047S-2081S.

22. Briend A, Maire B, Fontaine O et al. (2012) Mid-upper arm circumference and weight-for-height to identify high-risk malnourished under-five children. Matern Child Nutr $\mathbf{8}$, 130-133.

23. Myatt M, Khara T \& Collins S (2006) A review of methods to detect cases of severely malnourished children in the community for their admission into community-based therapeutic care programs. Food Nutr Bull 27, Suppl. 3, S7-S23.

24. World Health Organization (2017) Guideline: Assessing and Managing Children at Primary Health-Care Facilities To Prevent Overweight and Obesity in the Context of the Double Burden of Malnutrition. Updates for The Integrated Management of Childhood Illness (IMCI). Geneva: WHO. 